\title{
What Should Be the Treatment Protocol in Cases of Second and Third Degree Hemorrhoids?
}

\author{
Ajay Lunawat • Neelam Charles • Vikrant Ranjan
}

Received: 12 April 2014 / Accepted: 27 April 2014 / Published online: 10 May 2014

(C) Association of Surgeons of India 2014

Recently, we were going through one of the very interesting articles published in Indian Journal of Surgery, titled "Comparative study of hemorrhoidectomy and rubber band ligation in treatment of second and third degree hemorrhoids in Kashmir" by Gagloo et al. [1]. In our routine academic review of reputed journal articles, we found it very promising as the incidence of hemorrhoid patients comprises a significant portion of OPD and IPD. This article drew our attention because of its simple study design and thorough review of literature. Though there were minor flaws without which this study would have been more impressive. Like,

1. Authors compared the second and third grade hemorrhoids only; so, there should not be any mention of grade $\mathrm{I}$ in table 1 and table 2 of group $\mathrm{R}$ and group $\mathrm{H}$, respectively.

2. In the result section, ten patients were found with relative contraindication to surgery/anesthesia (who underwent rubber band ligation), but there is no mention of grade of hemorrhoids or about the study group of hemorrhoids. This part of result needs clarification in statistics too, as these things can affect the outcome analysis.

3. In the discussion part, the referring author name should be same at all places to avoid confusion, particularly, regarding the reference no 16 "Hosch et al."

A. Lunawat $\cdot \mathrm{N}$. Charles $\cdot \mathrm{V}$. Ranjan

Department of General Surgery, Sri Aurobindo Medical College and

Post Graduate Institute, Indore, India

N. Charles

e-mail: neelamcharles@rediffmail.com

V. Ranjan

e-mail: dr.vikrant.ranjan@gmail.com

A. Lunawat $(\square)$

46, Bhagwandeen Nagar, Indore, M.P., India 452001

e-mail: drajay_lunawat@rediffmail.com
4. We expect authors to include more recent references and to avoid the printing errors for better presentation of their study.

Apart from the above mentioned minor issues, the overall presentation was impressive. The authors also described the adverse effects and the complications of both the procedures briefly, which strengthened the conclusion.

We would like to mention about the similar conclusion noted by Shanmugam et al. in their study titled "Rubber band ligation versus excisional hemorrhoidectomy for hemorrhoids" [2]. This systematic review confirms the long-term efficacy of hemorrhoidectomy at least for grade III hemorrhoids. Rubber band ligation (RBL) can be adopted as the choice of treatment for grade II hemorrhoids with similar results but without the side effects of excisional hemorrhoidectomy (EH) while reserving EH for grade III hemorrhoids or in cases with recurrence after RBL. However, they needed more robust study to make definitive conclusions.

At last, we can conclude that in our scenario, particularly at tertiary centers, the protocol should be to go for RBL for first line treatment in grade II hemorrhoids and grade III hemorrhoids (where surgery or anesthesia is contraindicated). EH should be recommended to grade III hemorrhoid or in cases of failure of RBL in grade II hemorrhoids.

\section{References}

1. Gagloo MA, Hijaz SW, Nasir SA, Reyaz A, Bakshi IH, Chowdary NA et al (2013) Comparative study of hemorrhoidectomy and rubber band ligation in treatment of second and third degree hemorrhoids in Kashmir. Indian J Surg 75(5):356-360

2. Shanmugam V, Thaha MA, Rabindranath KS, Campbell KL, Steele RJ (2005) Rubber band ligation versus excisional haemorrhoidectomy for haemorrhoids. Cochrane Database Syst Rev 3, CD005034 